Bull. Austral. Math. Soc.

VOL. 48 (1993) [187-194]

\title{
ON SOME $P$-ESTIMATES FOR BANACH SPACES
}

\author{
D. Kutzarova, E. Maluta and S. Prus
}

Relations between $l_{p}$-type estimates of Khamsi and a uniform version of the KadecKlee property are studied. Khamsi's result on normal structure is strengthened.

\section{INTRODUCTION}

The notion of normal structure was introduced by Brodskii and Milman in [1]. Let us recall that a Banach space $X$ has normal structure if every bounded convex subset $A$ of $X$ with $\operatorname{diam} A>0$ contains an element $x$ such that

$$
\sup \{\|y-x\|: y \in A\}<\operatorname{diam} A .
$$

This property turned out to have important applications in fixed point theory (see [5]) and many conditions were found which imply normal structure. One of them was defined in [8]. Namely for a Banach space $X$ let us put

$$
D(X)=\sup \left\{\limsup _{n \rightarrow \infty} \operatorname{dist}\left(x_{n+1}, \operatorname{co}\left\{x_{k}\right\}_{k=1}^{n}\right)\right\}
$$

where the supremum is taken over all sequences $\left(x_{n}\right)$ in $X$ with $\operatorname{diam}\left\{\boldsymbol{x}_{n}\right\}=1$. It was proved that if $D(X)<1$, then $X$ is reflexive and has normal structure [8]. Some classes of Banach spaces $X$ for which $D(X)<1$ were considered in [6].

Another geometric property stronger than normal structure was invented by Khamsi in [4]. The aim of this paper is to study relations between a property from [6] and that of Khamsi. As a consequence we shall see that for reflexive spaces Khamsi's property actually gives the condition $D(X)<1$.

\section{PReliminaries}

In [3] Huff introduced a generalisation of uniform convexity. He called it nearly uniform convexity.

A slight modification of a result from [3] shows that a Banach space $X$ is nearly uniformly convex if and only if $X$ is reflexive and for every $\epsilon>0$ there exists $\delta>0$

Received 10 August 1992

Copyright Clearance Centre, Inc. Serial-fee code: 0004-9729/93 $\$ A 2.00+0.00$. 
such that whenever $\left(x_{n}\right)$ is a sequence in the unit ball $B_{X}$ of $X$ with $x_{n} \stackrel{w}{\longrightarrow} x$ and $\inf \left\{\left\|x_{n}-x\right\|: n \geqslant 1\right\}>\epsilon$, then

$$
\|x\| \leqslant 1-\delta
$$

Spaces which satisfy the last condition are called uniformly Kadec-Klee spaces. In [2] weakly uniformly Kadec-Klee spaces were defined. The following version of that definition agrees with the one given in [6].

A Banach space $X$ is WUKK' provided there exist constants $\epsilon, \delta \in(0,1)$ such that if $\left(x_{n}\right)$ is a sequence in $B_{X}$ with $x_{n} \stackrel{w}{\longrightarrow} x$ and $\inf \left\{\left\|x_{n}-x\right\|: x \geqslant 1\right\}>\epsilon$, then

$$
\|x\| \leqslant 1-\delta
$$

In this paper we shall deal mainly with Banach spaces which have finite dimensional decompositions (FDD in short). Let us recall that a sequence $\left(X_{n}\right)$ oi finite dimensional subspaces of a Banach space $X$ is said to be an FDD of $X$ if every $x \in X$ has a unique representation of the form

$$
x=\sum_{k=1}^{\infty} x_{k}
$$

where $x_{k} \in X_{k}$, for all $k$.

The element $x \neq 0$ is called a block if the set $\operatorname{supp} x=\left\{k: x_{k} \neq 0\right\}$ is finite. The FDD $\left(X_{n}\right)$ is bimonotone whenever

$$
\max \{\|x\|,\|y\|\} \leqslant\|x+y\|
$$

for any two blocks $x, y$ with $\max \operatorname{supp} x<\min \operatorname{supp} y$.

The following definition is a modification of the condition which was considered in [4].

DEFINITION: A Banach space $X$ with an FDD has property (K) if there exist constants $p \in[1, \infty), \lambda \in(0,2)$ such that

$$
\|x\|^{p}+\|y\|^{p} \leqslant \lambda\|x+y\|^{p}
$$

for any two blocks $x, y \in X$ with $\max \operatorname{supp} x+1<\min \operatorname{supp} y$.

Analysis of the James space (see [4]) shows that the prcperty (K) is essentially weaker than that of Khamsi. However it is easy to see that all the results in [4] remain valid if one uses property $(K)$. 


\section{RESULTS}

Let $Y$ be a closed subspace of a Banach space $X$. We denote by $\operatorname{codim}_{X} Y$ the dimension of the quotient space $X / Y$.

Proposition 1. If $Y$ is a closed subspace of a Banach space $X$ with $\operatorname{codim}_{X} Y$ $<\infty$, then

$$
D(X)=D(Y) .
$$

ProOF: Let $Y$ be a closed subspace of $X$ with $\operatorname{codim}_{X} Y<\infty$. Clearly $D(Y) \leqslant$ $D(X)$.

In order to show the opposite inequality let us fix a positive $\epsilon<D(X)$ and choose a sequence $\left(x_{n}\right)$ in $X$ so that

$$
D(X)-\epsilon \leqslant \limsup _{n \rightarrow \infty} \operatorname{dist}\left(x_{n+1}, \operatorname{co}\left\{x_{k}\right\}_{k=1}^{n}\right)
$$

and $\operatorname{diam}\left\{x_{n}\right\}=1$.

By our assumption there exists a finite dimensional subspace $Z$ of $X$ such that $X=Y \oplus Z$. Therefore for each $n$ we have a decomposition $x_{n}=y_{n}+z_{n}$, where $y_{n} \in Y, z_{n} \in Z$. Moreover the sequence $\left(z_{n}\right)$ is bounded. Passing to a subsequence, we can assume that $\left\|z_{m}-z_{n}\right\| \leqslant \epsilon$ for all $m, n$. Consequently

$$
\left\|y_{n}-y_{m}\right\| \leqslant\left\|x_{n}-x_{m}\right\|+\left\|z_{m}-z_{n}\right\| \leqslant 1+\epsilon
$$

for all $m, n$. Hence $d=\operatorname{diam}\left\{y_{n}\right\} \leqslant 1+\epsilon$ and clearly $d>0$.

Let us put $y_{n}^{\prime}=(1 / d) y_{n}$. Then $\operatorname{diam}\left\{y_{n}^{\prime}\right\}=1$. Moreover for any nonnegative $\lambda_{1}, \ldots, \lambda_{n}$ with $\sum \lambda_{i}=1$ we have

$$
\begin{aligned}
\left\|y_{n+1}^{\prime}-\sum_{i=1}^{n} \lambda_{i} y_{i}^{\prime}\right\| & \geqslant \frac{1}{d}\left(\left\|x_{n+1}-\sum_{i=1}^{n} \lambda_{i} x_{i}\right\|-\left\|z_{n+1}-\sum_{i=1}^{n} \lambda_{i} z_{i}\right\|\right) \\
& \geqslant \frac{1}{d}\left(\operatorname{dist}\left(x_{n+1}, \operatorname{co}\left\{x_{i}\right\}_{i=1}^{n}\right)-\epsilon\right) .
\end{aligned}
$$

It follows that

$$
\underset{n \rightarrow \infty}{\limsup \operatorname{dist}}\left(y_{n+1}^{\prime}, \operatorname{co}\left\{y_{i}^{\prime}\right\}_{i=1}^{n}\right) \geqslant \frac{1}{d}(D(X)-2 \epsilon) .
$$

In view of the definition of $D(Y)$ this shows that

$$
D(Y) \geqslant \frac{1}{d}(D(X)-2 \epsilon) \geqslant \frac{1}{1+\epsilon}(D(X)-2 \epsilon) .
$$

Since $\epsilon>0$ may be arbitrarily small, we finally obtain

$$
D(Y) \geqslant D(X) \text {. }
$$


THEOREM 2. Let $X$ be a Banach space with an FDD. If $X$ has property $(\mathrm{K})$, then $X$ is WUKK'.

Proof: Let us assume that $X$ has property $(K)$ and let $p \geqslant 1, \lambda \in(0,2)$ be as in Definition 1.

Consider a sequence $\left(x_{n}\right)$ in $B_{X}$ with $x_{n} \stackrel{w}{\longrightarrow} x$ and $\inf \left\{\left\|x_{n}-x\right\|: n \geqslant 1\right\}>\epsilon$, where $\epsilon=(\lambda / 2)^{1 / p}$. Given $\eta>0$, we can find a block $u \in X$ for which $\|x-u\|<\eta$. Since the sequence $\left(x_{n}-x\right)$ converges weakly to zero, there exists an index $n$ and a block $v$ such that $\max \operatorname{supp} u+1<\min \operatorname{supp} v$ and $\left\|x_{n}-x-v\right\|<\eta$. By our assumption we have

$$
\|u\|^{p}+\|v\|^{p} \leqslant \lambda\|u+v\|^{p} .
$$

Therefore

$$
(\|x\|-\eta)^{p}+\left(\left\|x_{n}-x\right\|-\eta\right)^{p} \leqslant \lambda\left(\left\|x_{n}\right\|+2 \eta\right)^{p}
$$

It follows that

$$
(\|x\|-\eta)^{p}+(\epsilon-\eta)^{p} \leqslant \lambda(1+2 \eta)^{p}
$$

Passing to the limit with $\eta \rightarrow 0$, we finally get

$$
\|x\|^{p} \leqslant \lambda-\epsilon^{p} .
$$

Thus it suffices to put $\delta=1-(\lambda / 2)^{1 / p}$.

In the particular case of reflexive spaces the above results and [6, Theorem 4] give us the following strengthened version of Theorem 3 of [4].

COROLLARY 1. Let $Y$ be a closed subspace with an FDD of a reflexive space $X$. If $\operatorname{codim}_{X} Y<\infty$ and $Y$ has property $(\mathrm{K})$, then

$$
D(X)<1 \text {. }
$$

Now we turn to a result which is a partial converse of Theorem 2. For stating it we first need to recall the following two notions.

Let $\left(X_{n}\right)$ be an FDD of a Banach space $X$. A sequence $\left(Y_{n}\right)$ of finite dimensional subspaces of $X$ is called a blocking of $\left(X_{n}\right)$ if there exists an increasing sequence of integers $\left(n_{k}\right)$ with $n_{1}=0$ such that

$$
Y_{k}=X_{n_{k}+1} \oplus \cdots \oplus X_{n_{k+1}}
$$

for all $k$. Clearly the sequence $\left(Y_{k}\right)$ is an FDD of $X$.

An FDD $\left(X_{n}\right)$ of a space $X$ is shrinking (see [7] for the definition) if and only if every bounded sequence of blocks $\left(z_{n}\right)$, with $k \leqslant \min \operatorname{supp} z_{k}$ for all $k$, is weakly convergent to zero. This is the case for example if the space $X$ is reflexive. 
Proposition 3. Let $X$ be a Banach space with a shrinking FDD $\left(X_{n}\right)$. If $X$ is WUKK', then there exists a constant $c>0$ and a blocking $\left(Y_{k}\right)$ of $\left(X_{n}\right)$ such that

$$
1+c \leqslant\|x+y\|
$$

whenever $x, y \in X$ are two blocks with $\|x\|=\|y\|=1$ and $\max \operatorname{supp} x+1<\min \operatorname{supp} y$, where the supports are taken with respect to $\left(Y_{k}\right)$.

Proof: If $X$ is WUKK', then from the definition we obtain some constants $\epsilon, \delta \in$ $(0,1)$. If suffices to show that there exists a constant $c>0$ which satisfies the following property (compare to [9]). For every $n$ there is $m>n$ such that

$$
1+c \leqslant\|x+y\|
$$

whenever $\|x\|=\|y\|=1$ and $\max \operatorname{supp} x \leqslant n, m \leqslant \min \operatorname{supp} y$, where the supports are taken with respect to $\left(X_{n}\right)$.

Let us assume the contrary. Then for every positive $c<1 /(\max \{\epsilon, 1-\delta\})-1$ we can find an integer $n$ and two sequences of blocks $\left(x_{k}\right),\left(y_{k}\right)$ such that

$$
\left\|x_{k}+y_{k}\right\|<1+\frac{1}{2} c
$$

$\left\|x_{k}\right\|=\left\|y_{k}\right\|=1, \max \operatorname{supp} x_{k} \leqslant n$ and $k \leqslant \min \operatorname{supp} y_{k}$ for all $k$.

The sequence $\left(x_{k}\right)$ is contained in the unit sphere of a finite dimensional subspace of $X$. Consequently, passing to a subsequence, we can assume that it converges to some $\boldsymbol{x}$.

Let us consider a sequence of elements $z_{k}=(1+c)^{-1}\left(x+y_{k}\right)$. By our assumption it converges weakly to $z=(1+c)^{-1} x$. Moreover $\left\|z_{k}-z\right\|=(1+c)^{-1}>\epsilon$ for all $k$ and $\left\|z_{k}\right\| \leqslant 1$ for sufficiently large $k$. On the other hand $\|z\|=(1+c)^{-1}>1-\delta$, which contradicts the assumption that $X$ is WUKK'.

THEOREM 4. Let $X$ be a Banach space with a bimonotone shrinking FDD $\left(X_{n}\right)$. If $X$ is WUKK', then there exists a blocking $\left(Y_{k}\right)$ of $\left(X_{n}\right)$ such that the space $X$ with the FDD $\left(Y_{k}\right)$ has property $(K)$.

Proof: Let us assume that $X$ is WUKK' and let $\left(Y_{k}\right)$ and $c>0$ be as in Proposition 3. We shall prove that $X$ has a property which is even stronger than (K).

Let us fix an arbitrary $p \in[1, \infty)$ and two blocks $x, y$ such that max supp $x+1<$ min supp $y$, where the supports are taken with respect to $\left(Y_{k}\right)$. We shall show that

$$
\|x\|^{p}+\|y\|^{p} \leqslant\left(1+a^{p}\right)\|x+y\|^{p},
$$

where $a=(1+c)^{-1}<1$. 
To this end let us put $t=\|x\|, s=\|y\|, x^{\prime}=(1 / t) x$ and $y^{\prime}=(1 / s) y$. In the case when $t / s \leqslant a$ we apply the assumption that $\left(X_{n}\right)$ is bimonotone. Namely

$$
\|x+y\|^{p} \geqslant s^{p} \geqslant\left(t^{p}+s^{p}\right)\left(1+a^{p}\right)^{-1} \text {. }
$$

Let us in turn assume that $a<t / s \leqslant 1$. By Proposition 3 we obtain

$$
\begin{aligned}
\|x+y\|^{p} & =s^{p}\left\|\frac{t}{s} x^{\prime}+y^{\prime}\right\|^{p} \\
& \geqslant s^{p}\left(\left\|x^{\prime}+y^{\prime}\right\|-1+\frac{t}{s}\right)^{p} \geqslant s^{p}\left(\frac{1}{a}-1+\frac{t}{s}\right)^{p} \\
& \geqslant\left(\frac{t}{a}\right)^{p} \geqslant\left(t^{p}+s^{p}\right)\left(1+a^{p}\right)^{-1} .
\end{aligned}
$$

The remaining cases may be handled in a similar way.

The assumption that an FDD is bimonotone cannot be omitted. In general even nearly uniform convexity does not imply property (K).

EXAMPLE. Let us consider a norm in the plane given by the formula

$$
\|(\alpha, \beta)\|_{0}=\max \left\{\left|\frac{1}{4} \alpha+\beta\right|,\left(\alpha^{2}+\left(\frac{1}{2} \beta\right)^{2}\right)^{1 / 2}\right\}
$$

where $\alpha, \beta$ are real numbers.

By $X$ we denote the space $l_{2}$ with the following equivalent norm

$$
\|x\|=\sup \left\{\left(\left\|\left(\alpha_{1}, \alpha_{k}\right)\right\|_{0}^{2}+\left(\frac{1}{2}\left\|Q_{k} x\right\|_{2}\right)^{2}\right)^{1 / 2}: k>1\right\}
$$

where $x=\left(\alpha_{n}\right) \in l_{2}, Q_{k} x=\left(\alpha_{n}\right)_{n>k}$ and $\|\cdot\|_{2}$ is the $l_{2}$-norm.

We shall show that $X$ is nearly uniformly convex and does not have property (K) for any FDD. For this purpose let us fix $\epsilon>0$ and consider a sequence $\left(x_{n}\right)$ in $B_{X}$ weakly convergent to $x$ with $\inf \left\{\left\|x_{n}-x\right\|: n \geqslant 1\right\}>\epsilon$. Since $\left(x_{n}-x\right)$ converges weakly to zero, for every $\eta>0$ we can find an index $n$ and two blocks $u, v$ such that

$$
\|x-u\|<\eta,\left\|x_{n}-x-v\right\|<\eta
$$

and max supp $u<\min \operatorname{supp} v$, where the supports are taken with respect to the natural basis of $\boldsymbol{X}$. Clearly 


$$
\|u\|^{2}+\left(\frac{1}{2}\|v\|\right)^{2} \leqslant\|u\|^{2}+\left(\frac{1}{2}\|v\|_{2}\right)^{2} \leqslant\|u+v\|^{2} .
$$

Therefore

$$
(\|x\|-\eta)^{2}+\left(\frac{1}{2}\left(\left\|x_{n}-x\right\|-\eta\right)\right)^{2} \leqslant\left(\left\|x_{n}\right\|+2 \eta\right)^{2}
$$

and consequently

$$
(\|x\|-\eta)^{2}+\left(\frac{1}{2}(\epsilon-\eta)\right)^{2} \leqslant(1+2 \eta)^{2} .
$$

By passing to the limit with $\eta \rightarrow 0$, it follows that

$$
\|x\| \leqslant\left(1-\left(\frac{1}{2} \epsilon\right)^{2}\right)^{1 / 2} .
$$

This shows that the space $X$ is uniformly Kadec-Klee. Since $X$ is reflexive, it is also nearly uniformly convex.

Let us now assume that the space $X$ with some FDD has property (K) with constants $p \geqslant 1$ and $\lambda \in(0,2)$. We consider the elements

$$
x=\left(-\frac{4}{5}, 0, \ldots\right), \quad y_{n}=\left(0, \ldots, 0, \frac{6}{5}, 0, \ldots\right),
$$

where $6 / 5$ is the $(n+1)$ th coordinate. Straightforward computations show that $\|x\|=$ $4 / 5,\left\|y_{n}\right\|=6 / 5$ and $\left\|x+y_{n}\right\|=1$ for all $n$. Moreover the sequence $\left(y_{n}\right)$ converges weakly to zero. Therefore an argument similar to that in the proof of Theorem 2 gives

$$
\left(\frac{4}{5}\right)^{p}+\left(\frac{6}{5}\right)^{p} \leqslant \lambda
$$

which contradicts the assumption that $\lambda<2$.

Let us remark that, using an argument from [6], one can show that the space $X$ has so called property $(\beta)$ (see [10]) which is even stronger than nearly uniform convexity.

\section{REFERENCES}

[1] M.S. Brodskii and D.P. Milman, 'On the center of a convex set', Dokl. Akad. Nauk SSSR 59 (1948), 837-840.

[2] D. van Dulst and B. Sims, 'Fixed points of nonexpansive mappings and Chebyshev centers in Banach spaces with norms of type $\mathrm{KK}$ ', in Banach space theory and its applications (Springer-Verlag, Berlin, Heidelberg, New York, 1983), pp. 35-43. 
[3] R. Huff, 'Banach spaces which are nearly uniformly convex', Rocky Mountain J. Math. 10 (1980), 743-749.

[4] M.A. Khamsi, 'Normal structure for Banach spaces with Schauder decomposition', Canad. Math. Bull. 32 (1989), 344-351.

[5] W.A. Kirk and K. Goebel, Topics in metric fixed point theory (Cambridge University Press, Cambridge, 1990).

[6] D. Kutzarova, E. Maluta and S. Prus, 'Property $(\beta)$ implies normal structure of the dual space', Rend. Circ. Mat. Palermo (to appear).

[7] J. Lindenstrauss and L. Tzafriri, Classical Banach spaces I. Sequence spaces (SpringerVerlag, Berlin, Heidelberg, New York, 1977).

[8] E. Maluta, 'Uniformly normal structure and related coefficients', Pacific J. Math. 111 (1984), 357-369.

[9] S. Prus, 'Nearly uniformly smooth Banach spaces', Boll. Un. Mat. Ital. (7) 3-B (1989), 507-521.

[10] S. Rolewicz, 'On $\Delta$-uniform convexity and drop property', Studia Math. 87 (1987), 181-191.

Inst. of Mathematics

Bulgarian Acad. of Sciences

1090 Sofia

Bulgaria

Department of Mathematics

M. Curie-Skłodowska University

20-031 Lublin

Poland
Dip. di Matematica-Politecnico

via Bonardi 9

20133 Milano

Italy 